\title{
Rutgers University Team Takes Top Prize in 2021 e-Fest
}

\section{Brittney Quant (Opus College of Business)}

KEYWORDS: Innovation, student entrepreneurs, efest, Student innovators.

The winning Rutgers University team included (left to right) Juliet Petillo, Harrison Zhang, Clairisse Whang and Alyssa Krisinski

MINNEAPOLIS, April 26, 2021 - For the first time in its five-year history, e-Fest was held virtually in an interactive online format that brought together 25 Finalist teams for a three-day celebration of undergraduate entrepreneurship. Competitors featured inventors of medical devices, technologies, apps, retail services, social ventures, and more. So inspiring! Click here(https://eiexchange.com/content/announcingour-2021-efest-finalists) to learn more about this year's finalists. You can find more information about the next eFest competition here(https://efest.biz) .

We are very pleased to announce the results of the Schulze Entrepreneurship Challenge:

\section{Grand Champion, First Place - $\$ 50,000$}

Nutrivide, Rutgers University

Nutrivide is redefining nutrient and drug delivery for infants: through a device that functions like a pacifier, holding a cartridge containing the medicine. For centuries, advancements in nutrition and medicine taken orally have dominated the global scientific response to illness. However, health systems striving to offer treatment often fall short and for an entirely preventable reason: inaccurate dosing and squeamish infants. Nutrivide plans to address this problem directly and locally, beginning at the New York-Presbyterian Health System.

Team: Alyssa Krisinski, Juliet Petillo, Clairisse Whang, Harrison Zhang

Advisor: Mukesh Patel

\section{Runner-Up, Second Place - $\$ \mathbf{3 0 , 0 0 0}$}

\begin{abstract}
MassApply, Virginia Tech
MassApply is a suite of job hunt automation tools that can help someone land 10 times more interviews. The team has built a cold-email outreach tool that lets applicants connect directly with recruiters in a single click, and a CRM to help manage their job hunt. MassApply (massapply.com) has helped over 12,000 people so far and aims to support the 10 million professionals on the job hunt every year.
\end{abstract}

Team: Sana Ahmad \& Naman Singh

Advisor: Sean Collins

\section{Third Place - $\$ 20,000$}

Hydrova, Georgetown University \& Massachusetts Institute of Technology (MIT)

Hydrogen has incredible potential to combat climate change as a cleantech fuel, but the current process for making it is unsustainable. Hydrova has developed a disruptive method for producing clean and low-cost hydrogen using renewable materials: scrap aluminum and water. This method has the added value of enabling on-site and on-demand hydrogen production, avoiding the high costs of transporting hydrogen.

Team: Julian Davis \& Rostam Reifschneider

Advisor: Addison Stark

\section{Fourth Place - $\$ 15,000$}

Telo, University of Minnesota - Twin Cities

Telo has designed the first major innovation on the rollator walker in over 40 years. Until now, this device was largely unchanged since its creation in 1978, despite being something millions of people rely on every day. Telo's patent-pending design features a reverse frame that puts the user at its center, with integrated back support to prevent the device from getting ahead 
of them.

Team: Steven Bleau, Morgan Kerfeld, Rick Pradhan, Elizabeth Urbanski

Advisor: John Stavig

\section{Fifth Place - $\$ 10,000$}

Renter Chat, Northeastern University

Renter Chat is an Al-powered chatbot for apartment websites. The small chatbot window acts as an intelligent virtual leasing agent, assisting the renter with everything from answering questions to scheduling tours. Renter Chat leverages Google's Dialogflow natural language processing platform to provide consistent, human-like, Al-powered responses to common and unique client questions alike.

Team: Ryan Soderberg and Will Kofski

Advisors: Esther Chewning \& Max Rampulla

\section{Audience Choice - $\$ 1,000$}

Telo, University of Minnesota - Twin Cities

\section{Second Place, Room Finalist Teams (Each Team Winning \$2,500)}

Aeropest, Drexel University

Brave Virtual Worlds, University of Virginia

Cress Health, Hofstra University \& Brown University

Keyrift, Florida Gulf Coast University

Pill Skills, University of Wisconsin - Madison

\section{Third Place, Room Finalist Teams (Each Team Winning \$1,000)}

Boosted Chews, University of Wisconsin - Madison

EthioPay, Georgia State University

Mo.Na Gems, Johns Hopkins University

Stormie Seas, Florida Gulf Coast University

Virgo, University of Northern lowa
In addition to the winners of our Schulze Entrepreneurship Challenge, e-Fest recognized two teams with outstanding potential for Social Impact and Global Impact.

\section{Social Impact - $\$ 10,000$}

Cress Health, Hofstra University \& Brown University

Team: Michael Lai and Justin Kim

Advisor: Stacey Sikes

\section{Global Impact - $\$ 10,000$}

Nutrivide, Rutgers University

Team: Alyssa Krisinski, Juliet Petillo, Clairisse Whang, Harrison Zhang

Advisor: Mukesh Patel

e-Fest also includes two additional competitions - the Pitch Slam \& Innovation Challenge. Teams winning the Pitch Slam each receive $\$ 3,500$, and the winning Innovation Challenge teams receive $\$ 10,000$.

\section{Pitch Slam! - $\$ 3,500$}

Cress Health, Hofstra University \& Brown University

MELA, George Washington University

Nutrivide, Rutgers University

SPAITR, University of New Hampshire

Telo, University of Minnesota

\section{Innovation Challenge - $\$ 10,000$}

Teams are comprised by mixing students from different universities. Winning teams took home \$10,000 each, and the audience choice team won an additional $\$ 5,000$.

Group 1: Workspace - Aditya Parihar, Bradyen Esmaili, Jake Laxton, Clairisse Whang, Walter Malcolm

Group 2: ReSpace - Sean DeRossett, Evan 
Magnusson, Sophia Hardesty-Meteyer, Maddie

Palmersheim

Group 3: Immortal - Katherine Seunghyun Min, Naman Singh, Rostam Reifschneider, Will Kofski

\section{Audience Choice - $\$ 5,000$}

Workspace - Aditya Parihar, Bradyen Esmaili, Jake Laxton, Clairisse Whang, Walter Malcolm 